\title{
Nightmare Disorder
}

National Cancer Institute

\section{Source}

National Cancer Institute. Nightmare Disorder. NCI Thesaurus. Code C95076.

A sleep disorder characterized by the repeated occurrence of frightening dreams which precipitate awakenings from sleep; on awakening, the individual becomes fully alert and oriented and has detailed recall of the nightmare, which usually involves imminent danger or extreme embarrassment to the individual. 